Puis-je, en tant que médecin de premier recours ayant quatre ans de pratique continuer d'appliquer des bandages durcissants (gouttière plâtrée, plâtre circulaire) en Scotchcast et les facturer, même si le tarif indique une valeur intrinséque de 6 pour le secteur de la chirurgie et de la chirurgie pédiatrique? Je ne reviendrai pas sur le mode stupide de la non-facturation du matériel (un matériel bientôt plus cher que la prestation) ...

U. Z. $\dot{a} B$.

La répartition en secteurs n'a rien à faire avec la qualification nécessaire de l'exécutant. Elle ne sert qu'à calculer le nombre de points de la prestation technique pour cette prestation. Quant à votre appréciation des forfaits pour le matériel, la Chambre médicale a pris une décision dans votre sens en la matière.
Le TarMed se place au-dessus du Code des obligations: à ma connaissance, le médecin travaille dans une relation de mandat selon l'art. 394 CO. L'art. 402 dit que le mandant doit rembourser au mandataire les dépenses et les intérêts faits par celui-ci pour l'exécution régulière $d u$ mandat et le libérer ainsi des obligations par lui contractées. Qu'en est-il dès lors des $200 \mathrm{fr}$. de matériel qui tout à coup doivent être compris dans la prestation? Je le répète, le TarMed veut primer le CO.

M. D. $\dot{a} M$.

La Chambre médicale a pris une décision selon laquelle le chapitre 21 des interprétations générales doit être adapté à la pratique par la délégation FMH aux négociations.

\section{Psychiatrietarif und Solidarität unter Ärzten}

\author{
A. Stucki
}

Mit seinem Artikel erwähnt R. Bleuler [1], dass die Psychiater in den Tarifverhandlungen nicht genügend ernst genommen werden und verlangt mehr Solidarität der Ärzte unter sich. Sein Anliegen ist berechtigt und keineswegs neu. Ein kleiner Rückblick auf Tarifverhandlungen im Kanton Bern vor bald 40 Jahren möge dies etwas beleuchten.

Nur wenige der jungen Kollegen wissen vermutlich, dass im Kanton Bern bis Mitte der sechziger Jahre die Krankenkassen psychiatrische Bemühungen nicht vergüteten. Als ich 1958 meine Praxis eröffnete, waren psychiatrische Leistungen im Kassentarif nicht erwähnt. Der Psychiater konnte lediglich mit der Konsultationstaxe von Fr. 5.- und bei einzelnen Kassen mit einem freiwilligen Beitrag etwa in der gleichen Höhe rechnen. Dem Patienten durfte aber zusätzlich privat Rechnung gestellt werden.

Damit bestand praktisch eine Zwei-KlassenMedizin sowohl für den psychiatrisch tätigen Arzt wie auch für den Patienten. Vielfach konnten sich nur etwas begüterte Patienten eine eingehende Behandlung bei einem Psychotherapeuten leisten. Aber es konnte damit auch zwei Klassen von Ärzten geben:

Korrespondenz:

Dr. med. Alfred Stucki

Bellevuestrasse 7a

CH-3600 Thun solche, die ihre Kundschaft nach deren finanziellen Möglichkeiten auswählten, während die andern die Indikation für eine systematische Psychotherapie allein nach medizinischen Kriterien stellten - sich dabei aber meist mit einem eher beschämend tiefen Einkommen zufriedengeben mussten. Das RobinHood-Prinzip, das noch unser Lehrer Jakob Klaesi empfahl (ein sehr reicher Patient sollte uns die Behandlung eines bedürftigen Hilfesuchenden finanzieren), war wohl nur ausnahmsweise praktikabel, und einem Arbeiter für Psychotherapie jeden Monat eine Rechnung in der Höhe von mindestens einem Drittel seines Monatsverdienstes zu stellen, verbot sich in den meisten Fällen.

Deshalb bekam ich nach dem ersten Jahresabschluss von meinem Buchhalter die Warnung, meine Praxis stehe schlecht (trotz 50 Stunden Konsultationen pro Woche), und die wachsende Familie war immer noch auf Zuschüsse von Eltern und Schwiegereltern angewiesen, wollte sie in noch eben «standesgemässen» Verhältnissen leben. Meinen Kollegen mag es vielfach ähnlich ergangen sein, und deshalb bildeten Urs Minder, Arthur Trenkel, Fritz Jaggi und ich eine Arbeitsgruppe, die schliesslich zur Gründung der kantonalen bernischen Fachgesellschaft (KBP) führte. Sie sah ihre dringendste Aufgabe darin, mit den Kassen einen Tarif für psychiatrische Leistungen auszuhandeln.

Ein Haupthindernis entstand für uns im Unverständnis und Misstrauen bei unseren Diskussionspartnern. Aber auch von den Kollegen anderer Fachgebiete sahen wir uns im Stich gelassen; ihnen wie den Kassenvertretern schien weitgehend das Verständnis dafür abzugehen, was Psychotherapie ist, und dass sie vernünftig entschädigt werden muss, sollte der Therapeut davon leben können. Den Psychiater in der Praxis sah man als plaudernden Onkel, der vor allem gelangweilten und sexuell 
unbefriedigten Frauen beistand. Auch nach grosser Aufklärungsarbeit unsererseits verstieg sich ein Kassenfunktionär zum Ausspruch, die wirtschaftlichste Lösung wäre wohl die Anstellung eines Kassenbocks (sic!), eines potenten Mannes, der sich frustrierter Frauen anzunehmen hätte. Ferner hatte man bei den Krankenkassen gelernt, dass Psychotherapie bisweilen auch Analyse genannt wurde, was dazu führte, dass man sie im Tarif der chemischen Laboranalysen ("Analysentarif") unterbringen wollte! So mussten sich zwangsläufig die Verhandlungen in die Länge ziehen.

\section{Erster Tarif für psychiatrische Leistungen}

Besonders bereitete es Kopfzerbrechen, nach welchen Massstäben die Entschädigung zu erfolgen hätte. Wir schlugen einen Zeittarif vor, wie er auch einer nach wie vor privat zu verrechnenden psychoanalytischen Langzeittherapie zugrunde liegen würde. Naiverweise nahmen wir an, dies müsste den Kassen am ehesten einleuchten, da die Verrechnung der Zeit für sie am leichtesten kontrollierbar wäre. Merkwürdigerweise wollte die Gegenseite hier nicht anbeissen, und zu unserer Verwunderung mussten wir erfahren, dass ausgerechnet unsere Kollegen anderer Sparten sich dagegen zu Wehr setzten. Der Grund war klar: Die meisten von ihnen hatten pro Stunde wesentlich höhere Einnahmen als wir mit den 50 Franken, die Mitte der sechziger Jahre für uns zu erwarten gewesen wären. Solche Ungleichheit hätte sich aber ungünstig auf ihren Tarif auswirken können.

Weil nun die Kassen darauf drängten, dass wir unsere psychiatrischen Leistungen definierten, kam Dr. Jaggi, der nachmalige Präsident der kantonalen Ärztegesellschaft, auf die Idee, einen Katalog von Lei- stungen in vier Stufen, von "leicht» bis "ausserordentlich schwierig" vorzuschlagen, unterteilt in diagnostische und therapeutische Massnahmen. Die Aussagekraft war sehr gering, das wusste Jaggi selbst am besten, doch wurde der Vorschlag rasch akzeptiert, unter der Bedingung, dass wir uns dabei nie auf den Zeitaufwand beziehen würden. Unter uns galt indessen die stillschweigende Übereinkunft, dass z.B. Position c) "komplexe aufdeckende Psychotherapie» etwa einem Zeitaufwand von 50 Minuten entsprechen sollte. Man hielt sich an diese Richtlinien, wenn auch "schwarze Schafe» die Möglichkeit gehabt hätten, die gleiche Leistung in 10 Minuten zu verrechnen.

So wurde 1967 ein brauchbarer Psychiatrietarif in Kraft gesetzt, der bei vernünftiger Anwendung um 50 Franken pro Stunde bot, was heute etwa Fr. 150.- entspricht. Damit war der Psychiater frei, unbeeinflusst von der finanziellen Potenz seiner Patienten zu praktizieren.

In den letzten Jahrzehnten dürfte das Verständnis für psychiatrische Bemühungen gewachsen sein und damit die Bedingungen gegeben, die Ärzte dieses Fachs als gleichwertige Kollegen $\mathrm{zu}$ respektieren. Dass chirurgisch und sonstwie invasiv tätige Ärzte mit Rücksicht auf sehr lange Weiterbildung und auch grössere Risiken immer noch viel besser verdienen, sei ihnen zugestanden. Der Unterschied sollte aber doch nicht zu gross werden, und es stünde den Kollegen, die sich des Mehrfachen eines durchschnittlichen Psychiatereinkommens erfreuen, gut an, die Seelenärzte in ihren Anliegen zu unterstützen.

\section{Literatur}

1 Bleuler R. GRAT/TarMed - Psychiatrie - Solidarität. Schweiz Ärztezeitung 2000;81(24):1308. 